\title{
Octahedral slip in nickel single crystals induced by scratch testing
}

\author{
E. A. Alfyorova ${ }^{1, \uparrow}$, D. V. Lychagin ${ }^{2}$, A. V. Filippov ${ }^{3}$ \\ †katerina525@mail.ru \\ ${ }^{1}$ National Research Tomsk Polytechnic University, 30 Lenina ave., Tomsk, 634050, Russia \\ ${ }^{2}$ National Research Tomsk State University, 36 Lenina ave., Tomsk, 634050, Russia \\ ${ }^{3}$ Institute of Strength Physics and Materials Sciences SB RAS, 2/4 Akademicheskii ave., Tomsk, 634050, Russia
}

\begin{abstract}
Investigation of the effect of crystallographic orientation on mechanical properties, behavior under loading, or deformation relief of single crystals is topical. One of the methods that make it possible to achieve unambiguous mutual orientation of the material structure and the applied load is scratch testing. This research is aimed at establishing the features of the deformation relief formation under various crystallographic conditions (orientation of the plane and scratch direction). The paper presents the experimental results on scratch testing on single crystals of nickel. The face of a single crystal (010) and two scratch directions [001], [101] were investigated. The scratch testing parameters at which slip begins on the $\{111\}$ planes have been determined. It is shown that the slip is activated most easily in the systems parallel to the direction of the indenter movement and in the systems oriented thereto at an angle of $45^{\circ}$. Three stages are identified on the scratch friction force vs normal load (F-N curve) and the correlation of the stages with the beginning of the shear traces formation is shown. The parameters of the transition to the third stage of the F-N curve and the magnitude of the friction force for scratch testing depend on the crystallographic orientation direction of the indenter movement.
\end{abstract}

Keywords: scratch testing, deformation relief, FCC single crystal.

\section{Introduction}

The study of the influence of the crystallographic orientation of single crystals on their behavior under loading, the deformation relief, and mechanical properties is a classic issue and is still topical. At the present time, there are many experimental and theoretical studies in which the correlation of crystallography with the features of deformation processes and the forming deformation relief is shown [1-11]. Despite this, in conducting a full-scale experiment, researchers inevitably face the problem of unambiguous mutual orientation of the material structure and the applied load. A scratch testing is one of the available methods that assist solving this problem. This method is used to study the physical and mechanical properties of solids, polymers, films, coatings, etc.

The data obtained with the scratch testing on single crystals and polycrystals of various materials are described in [12-18]. For single crystals, the ratio of crystallographic parameters with the penetration depth of the indenter, misorientations in the implantation zone, and a number of other parameters were established. Strong anisotropic effects were revealed during indentation and sliding with different orientations on certain face-centered cubic (FCC), bodycentered cubic (BCC), and hexagonal close-packed (HCP)structured single crystals and metallic polycrystalline [13].

The mechanical properties for surfaces (111), (001) and (110) of Au single crystals are quantitatively studied. It was found that the elastic indentation modulus for surface (111) is $36 \%$ larger than for (001) and 3\% larger than for surface (110).
For each orientation, plastic deformation occurred when the maximum resolved shear stress reached approximately 1.8 GPa on all slip planes $\{111\}$ [16].

Molecular Dynamics MD simulation of indentation scratching was performed on single crystal aluminum in different orientations and scratch directions, to investigate the extent of anisotropy in hardness and friction coefficient of this material [15].

In general, the scratch test method makes it possible to identify the features of physical processes depending on the crystallographic orientation.

The aim of the work is to establish the features of the deformation relief formation under various crystallographic conditions (the plane orientation and scratch direction).

\section{Material and technique}

Experimental studies are carried out on single crystals of nickel using a CSM Instrument macro scratch tester. It is assumed that the normal load increases linearly from 0.5 to $3 \mathrm{~N}$. The scratch length is $2 \mathrm{~mm}$. The scratch rate is constant and equal to $0.5 \mathrm{~mm} / \mathrm{min}$.

The deformation relief formed on the single crystal face (100) with the scratch directions [001], [101] was studied. These orientations and directions were chosen for the purpose of detailed investigation of the crystallographic direction of the effect of scratches on the plastic deformation processes under the action of an indenter. The deformation relief is examined with an Olympus LEXT 4100 laser scanning microscope. 


\section{Results and discussion}

Fig. 1 shows the optical image of the deformation relief formed after scratching the lateral face (100) in the [001] direction, the shear traces begin to appear at a normal force of $1.7 \mathrm{~N}$, the orientation of the shear traces (Fig. 1a) corresponds exactly to the theoretical crystallographic scheme presented in Fig. 1b. Traces are oriented at an angle of $45^{\circ}$ to the trajectory of scratching. In this case, the slip traces along the outputs of all four octahedral planes are observed. In uniaxial compression along the [001] direction, shear systems in these planes have the same Schmid factor 0.41 . When considering the cross section in a direction perpendicular to the shear traces, shear steps are clearly observed, the slip height is $140 \pm 3.2 \mathrm{~nm}$. For nickel this corresponds to the exit to the surface of about 560 dislocations (the Burgers vector for nickel $b=0.249 \mathrm{~nm}$ ).

The analysis of the results obtained on the face (100) in the scratch direction $[1-10]$ showed that when the force of $2.6 \mathrm{~N}$ is reached, the shear traces perpendicular to the scratch direction appear (Fig. 2a). This direction corresponds to the exit of the close-packed plane to the face (100) (Fig. 2b), but the shear traces are observed only on one side of the scratch. When a magnitude of the normal force is about $3 \mathrm{~N}$, shear traces begin to form on both sides of the scratch (Fig. 2c). The test is completed at this load, and in Fig. 2c, a trace from the exit of the indenter is observed. At the same time, somewhat earlier, at a normal force of about $2 \mathrm{~N}$, the formation of shear traces of shear begins parallel to the scratch direction (Fig. 2d).

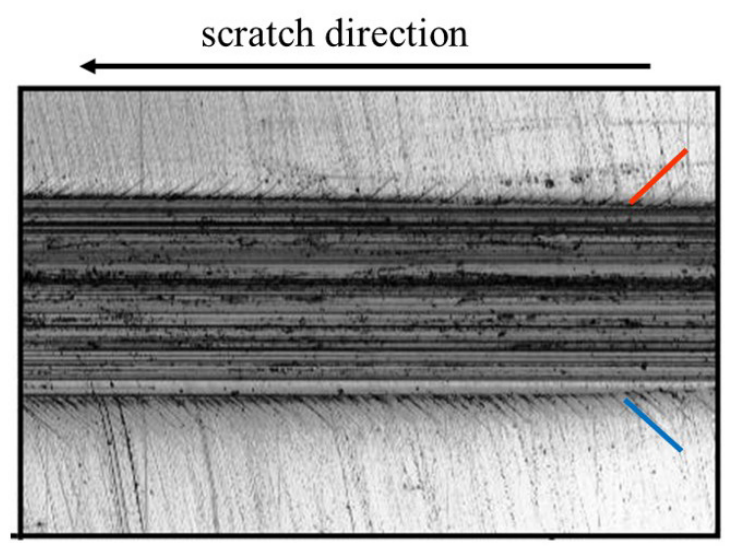

a

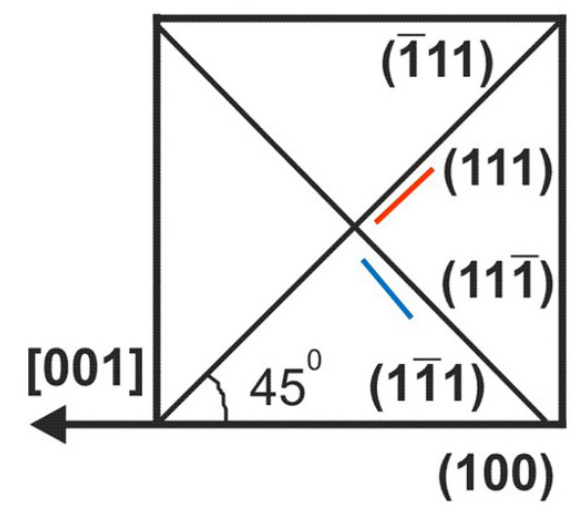

b

Fig. 1. The shear traces on the face (100) in the scratch direction [001] (a) and the scheme of the octahedral plane (b).
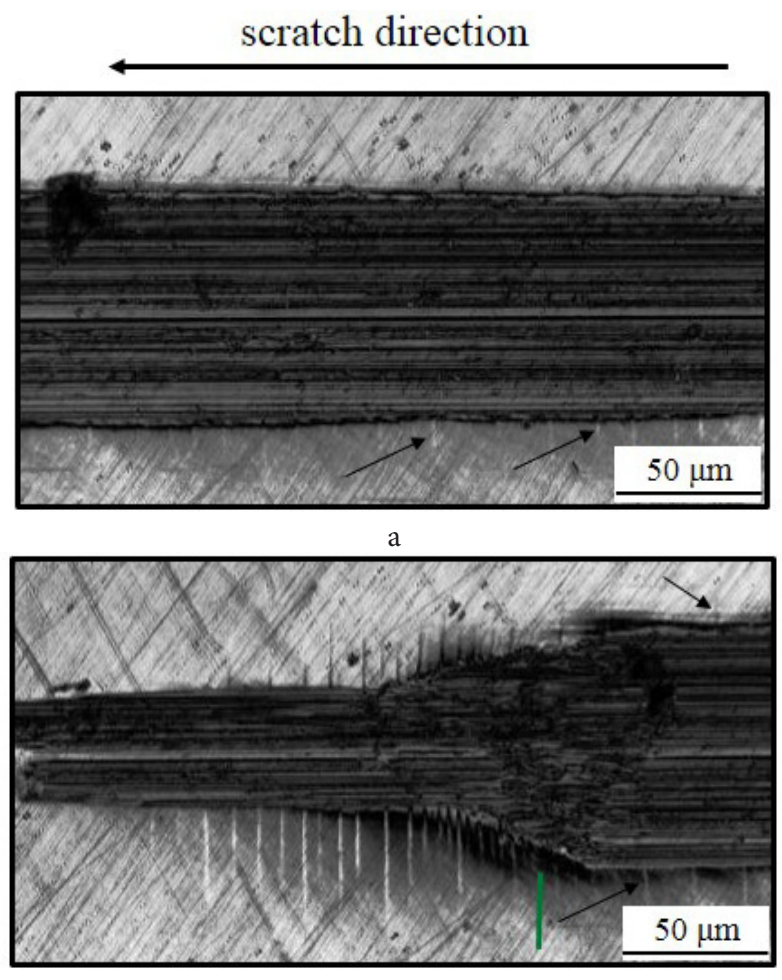

c

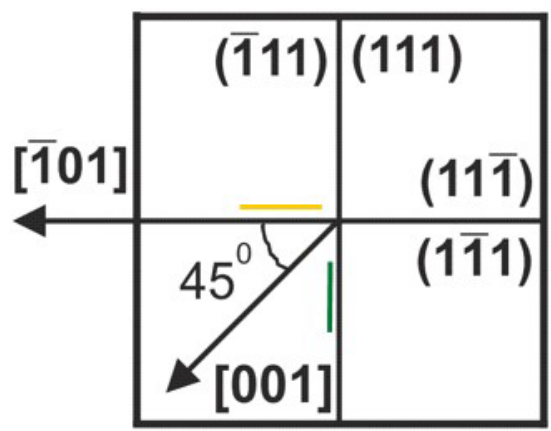

(100)

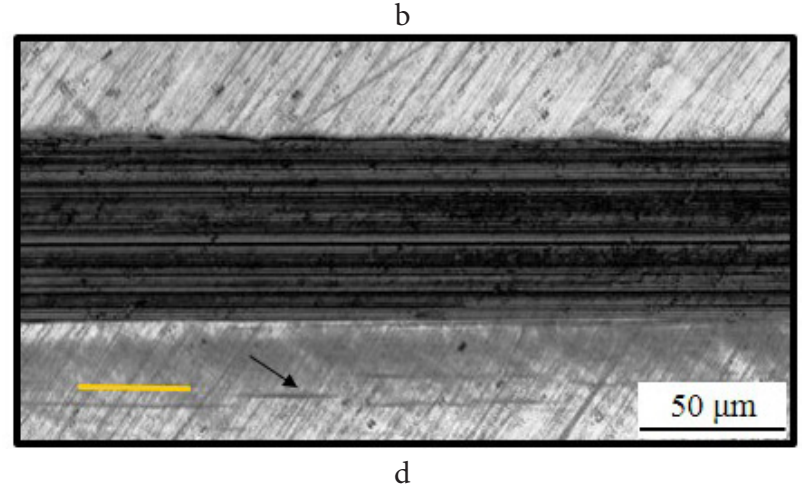

Fig. 2. The shear traces (indicated by arrows) on the face (100) in the scratch direction [110] at different loads (a, c, d) and the scheme of the octahedral plane (b). 
However, the complex nature of the stress field under the indenter also affects this formation. Discussion of this issue is beyond the scope of this paper. In [15], using the molecular dynamics method for aluminum single crystals, dislocations were observed in both parallel and perpendicular directions to the direction of indentation in the case of orientation [110] (110). On the contrary, dislocations were observed for propagation only parallel to the direction of indentation in the case of orientation [ $\overline{1} 10](001)$. In other cases, they are usually oriented at an angle to the scratch direction, which depends on the orientation of the crystal and the direction of the scratching.

Fig. 3 shows the dependence of the change of the penetration depth of the indenter on the normal load during the scratching process. The penetration depth of the indenter can be considered as a measure of the resistance to shear deformation during scratching, the smaller penetration depth of the indenter corresponds to greater resistance to deformation. When the face (100) is loaded in the direction of [001] and [ 101$]$, scratching in the direction [ $[101]$ results in a smaller penetration depth.

The friction force vs normal load (F-N curve) in Fig. 4 shows that the friction force of the scratch increases with increasing normal loads. This curve F-N can be divided into three stages: at the first stage, under normal loads from $0.5 \mathrm{~N}$ to $0.9 \mathrm{~N}$, the frictional force barely changes; at the second stage, from $0.9 \mathrm{~N}$ to $2.3 \mathrm{~N}$, the tangential resistance encountered by the scratch tip gradually increases; then, when the normal load becomes even larger, the frictional force increases significantly. These three stages are well correlated with three scratches, i.e., sliding along asperities, elastic deformation and a plastic groove, and also with the formation of shear traces on the surface. This process is typical for both cases. However, with the scratch direction [001], the frictional force is higher.

A similar separation in stages is described in [14] using the example of polycarbonate. At the same time, it is interesting to note that in the case of scratching along the [001] direction, the magnitude of the friction force is higher for the same normal force. This may be due to the fact that the penetration depth of the indenter in this case is greater (Fig. 3). Consequently, this crystallographic orientation is more plastic and the transition to the third stage occurs somewhat earlier than for the direction [101] (Fig. 4). Dependence of the penetration depth of the indenter and hardness on the crystallographic orientation of copper single

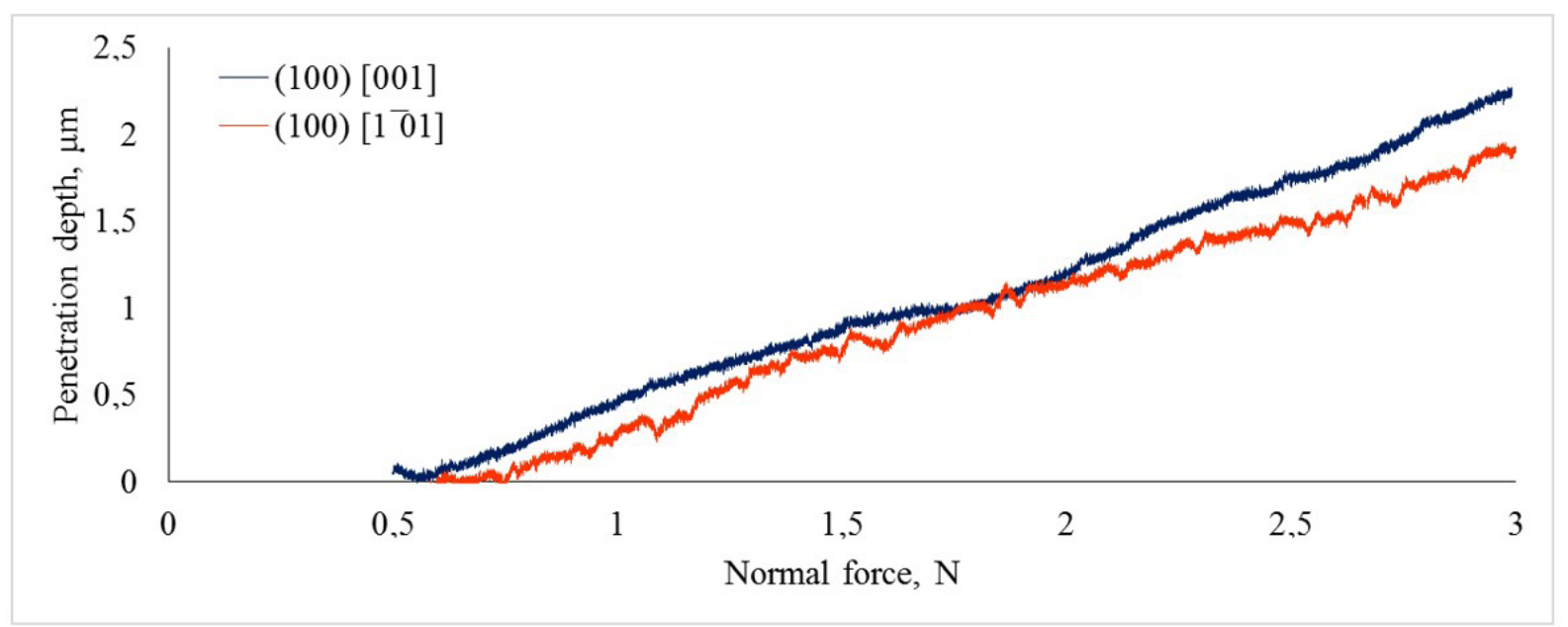

Fig. 3. (Color online) Penetration depth as function of normal loading for different crystallographic orientation of the nickel single crystals and scratch direction.

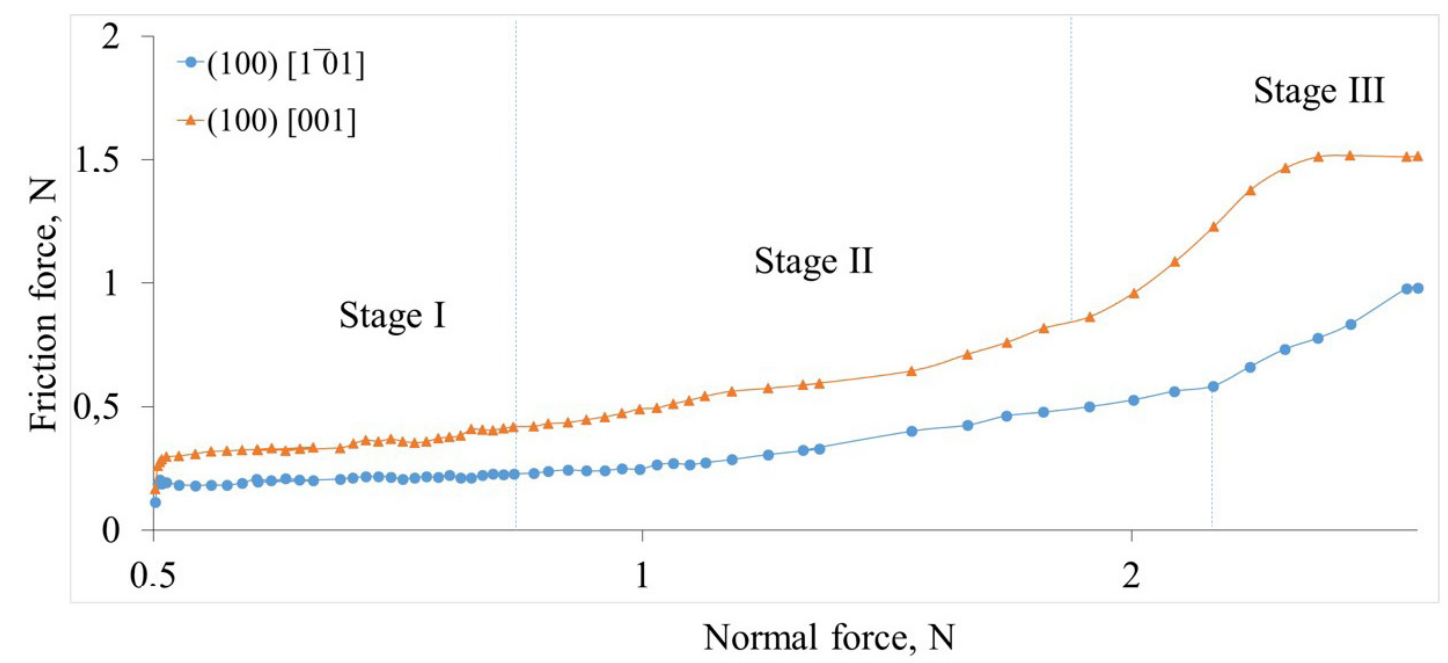

Fig. 4. (Color online) The scratch friction force vs normal load (F-N curve). 
crystals is discussed in [12], both experimental and modeling results are presented. In [15], similar results obtained by modeling for single crystals of aluminum are discussed.

\section{Conclusions}

Thus, for the variants of the crystallographic orientation of nickel single crystals considered in the present paper, the shearing along the close-packed planes is most easily activated in systems parallel to the direction of the indenter motion and in systems oriented at an angle of $45^{\circ}$. It is established that the shear strain resistance is higher for the scratch direction [001]. In both cases, on the F-N curve (Fig. 4), three stages can be distinguished. These stages correlate with the start of the formation of shear traces. The transition to the third stage occurs somewhat earlier for the scratch direction [001] than for the direction [ $\overline{101}$.

Acknowledgements. The results of laser scanning microscope have been obtained with the financial support of the Russian Foundation for Basic Research, according to the research project No. 16-32-60007 mol_a_dk. The preparation of samples and the results of indentation tests have been obtained with the financial support of Fundamental Research Program of State Academies for 2013-2020 (project No III.23.2.4).

\section{References}

1. M. Cai, S. C. Langford, J. Thomas Dickinson. Acta Mater. 56, 5938 (2008). DOI: 10.1016/j.actamat.2008.08.015

2. J. N. Florando, M.M. LeBlanc, D.H. Lassila. Scr. Mater. 57, 537 (2007). DOI: 10.1016/j.scriptamat.2007.05.014

3. W.Z. Han, G.M. Cheng, S.X. Li, S.D. Wu, Z.F. Zhang. Phys. Rev. Lett. 101, 3 (2008). DOI: 10.1103/PhysRevLett.101.115505
4. C. Ambrosi P., Schwink ChPias. Scr. Met. 12, 303 (1978).

5. K. R. Magid, J. N. Florando, D. H. Lassila, M. M. LeBlanc, N. Tamura, J. W. Jr Morris. Philos. Mag. 89(1), 77 (2009). DOI: 10.1080/14786430802558577

6. D. V. Lychagin, E.A. Alfyorova, V.A. Starenchenko. Phys. Mesomech. 14, $66 \quad$ (2011). DOI: 10.1016/j.physme.2011.04.009

7. D. V. Lychagin, S.Y. Tarasov, A.V. Chumaevskii, E.A. Alfyorova, Int. J. Plast. 69, 36-53 (2015), doi:10.1016/j.ijplas.2015.01.007.

8. D. V. Lychagin, E.A. Alfyorova, A.S. Tailashev. Russ. Phys. J. 58, 717 (2015). DOI: 10.1007/s11182-015-0556-Z

9. S. Ha, K. Kim. Math. Mech. Solids. 16, 652 (2011). DOI: $10.1177 / 1081286510387719$

10. I. Kireeva, Y. Chumlyakov, Z. Pobedennaya, D. Kuksgauzen, I. Karaman, H. Sehitoglu. AIP Conf. Proc. 1783, 1 (2016). DOI: 10.1063/1.4966383

11. I. Karaman, H. Sehitoglu, H.J. Maier, Y.I. Chumlyakow. Acta Mater. 49, 3919 (2001). DOI: 10.1016/S1359-6454(01)00296-8

12. M. Liu, K.A. Tieu, K. Zhou, C.T. Peng. Metall. Mater. Trans. A Phys. Metall. Mater. Sci. 47, 2717 (2016). DOI: $10.1007 / \mathrm{s} 11661-016-3439-1$

13. D.G. Flom, R. Komanduri. Wear. 252, 401 (2002). DOI: $10.1016 /$ S0043-1648(01)00879-1

14. J. Liu, H. Jiang, Q. Cheng, C. Wang. Tribol. Int. 125, 59 (2018). DOI: 10.1016/j.triboint.2018.04.024

15. R. Komanduri, N. Chandrasekaran, L. M. Raff. Wear. 240, 113 (2000). DOI: 10.1016/S0043-1648(00)00358-6

16. J. Kiely, J. Houston. Phys. Rev. B. 57, 12588 (1998). DOI: 10.1103/PhysRevB.57.12588

17. D. V. Lychagin, A. V. Filippov, O.S. Novitskaya, A. V. Kolubaev, O. V. Sizova. AIP Conf.Proc. 1909, 1 (2017). DOI: $10.1063 / 1.5013806$

18. A. Danyuk, D. Merson, A. Vinogradov. Vector of science of Togliatti State University. 3, 144 (2013). (in Russian) 